Reflexión y opinión

\title{
La Teoría del Capital Humano y su incidencia en la Educación. Un análisis desde la perspectiva mexicana
}

\section{The Human Capital Theory and its Impact on Education. An analysis from the Mexican perspective}

\author{
Silva Payró, Martha Patricia ${ }^{1}$; García Martínez, Verónica ${ }^{1}$ y Ramón Santiago, Pedro ${ }^{1}$
}

\begin{abstract}
Resumen:
El presente trabajo es una propuesta reflexiva sobre la Teoría del Capital Humano y su relación con la educación, tema que ha sido abordado desde diferentes perspectivas, sobre todo la económica, y posteriormente la sociológica. Se expone de manera sucinta la evolución de esta perspectiva teórica para coincidir en su injerencia con la educación. Se abordan además algunas consideraciones del caso de México. El objetivo fue analizar la manera en que el Capital Humano está invariablemente relacionado con la educación y la formación académica. Se concluye que el crecimiento económico de la persona depende no sólo de la educación recibida, sino de otros elementos ajenos al mismo individuo, así como que la concepción de que existe una relación directa proporcional entre educación y crecimiento económico ha cambiado a través del tiempo, y que una mayor formación educativa no garantiza el éxito.
\end{abstract}

Palabras Clave: Capital humano, desarrollo económico y social, Calidad de vida, Educación y desarrollo, México.

${ }^{1}$ Universidad Juárez Autónoma de Tabasco

*Correspondencia: patypayro@gmail.com
Keywords: Human capital, economic and social development, Quality of life, Education and Development, Mexico. 
La Teoría del Capital Humano (TCH) es una perspectiva economicista que ha madurado a lo largo de décadas y se ha robustecido de las aportaciones vertidas desde diferentes perspectivas disciplinares. Su planteamiento medular es el rendimiento que el recurso humano puede aportar a la organización y a la postre a la situación pecuniaria tanto del propio individuo como de la entidad donde se inserta. Este corpus teórico se alinea con la llamada economía del conocimiento, donde este último se convierte en un instrumento generador de riqueza.

El conocimiento adquirido mayormente a través de la educación, llega a ser para muchas personas la única manera de acceder a mejores condiciones de vida y a la movilidad social. Debido a esto la importancia de la $\mathrm{TCH}$ en la educación es inminente en los trabajos que ya existen al respecto (Labraña, Brunner y Álvarez, 2019, Quintero, 2020, Sassha, 2011, Tovar, 2017).

La Organización para la Cooperación y Desarrollo Económico [OCDE] (2007), señala al Capital Humano como el detonador del conocimiento que posee, desarrolla y acumula cada persona en su trayectoria de formación, así como la laboral y organizacional. De igual manera, hace un señalamiento en el sentido de que son escasas las Instituciones de Educación Superior (IES) que trazan en sus objetivos primarios, la contribución al desarrollo de la región en la que se sitúan. De modo que, frente a la necesidad de progreso económico, el individuo se puede topar con una oferta educativa y de formación que no satisface el mercado, las demandas sociales y su propia urgencia de escalar mejores niveles.

En México, como en el resto de los países de Latinoamérica, la educación se convierte en el puente entre la pobreza y riqueza de capital. El indicador relativo al análisis comparativo de México y su posicionamiento con la OCDE respecto a la inversión en ciencia (GIDE/PIB\%), señala que México está por debajo del promedio entre los países de esta organización que fue de $2.38 \%$ en 2014 . Como ejemplo, Corea invierte ocho veces más que México, y los países como Alemania, Estados Unidos o Japón, líderes en actividades científicas y tecnológicas, invierten por encima del promedio de la OCDE. Canadá, Francia y Reino Unido, aunque están por debajo del promedio, invierten entre tres y cuatro veces más que México (CONACYT, 2017). De manera que la baja inversión en generación del conocimiento puede estar incidiendo en la formación de Capital Humano.

Frente a este panorama, resulta necesario desarrollar estudios y análisis que permitan avanzar hacia la comprensión de la manera en que la educación puede estar ligada a la $\mathrm{TCH}$, en virtud de que ambas (educación y teoría) tienen como eje sustancial la transformación y mejoramiento del individuo a través del conocimiento. En el presente trabajo se expone en un primer momento la perspectiva teórica del Capital Humano y algunos antecedentes. Posteriormente se enuncian los aportes de este enfoque particularmente en la educación. En una última parte se exploran algunas condiciones observadas en México.

Con este análisis se pretende dilucidar cómo la educación y la formación académica pueden ser un parteaguas para el escalamiento social y económico de los sujetos, aunque no necesariamente, y convertirse en una especie de anomalía teórica. El impacto que la educación o la formación puede tener en la vida del individuo depende no sólo de su voluntad o de su preparación, sino de las condiciones que lo rodean. El tema es relevante porque representa la oportunidad de profundizar en un asunto poco explorado por su complejidad, y 
que puede dar cuenta de los altibajos que pueden ser parte de la trayectoria educativa de un individuo dentro del embalaje de la $\mathrm{TCH}$.

\section{Perspectiva teórica y conceptual de la TCH}

La TCH ha tenido impacto no solo en el ámbito educativo, esto puede advertirse a través de las aportaciones efectuadas por diversos autores a la Teoría del Capital Humano entre las que se destacan los componentes relativos a la ciencia, la investigación, la formación profesional, la situación laboral y las habilidades requeridas por los individuos. De esto se expone de manera sucinta lo más relevante.

Según Senior (1836) las habilidades de los trabajadores deben influir positivamente en su productividad y refuerza la idea de que la educación es el elemento indispensable para el desarrollo de los individuos. De igual manera, hizo mención del término de prestigio social que reciben los trabajadores preparados, quienes incurren en ciertos gastos derivados de su preparación, en espera que reporten beneficios a futuro, de tal forma que, en vez de ser vistas como decisiones de consumo, son decisiones de inversión.

Posteriormente, Stuart-Mill (1864), señaló que la productividad del trabajo está limitada por el conocimiento que tienen los trabajadores, y esto en conjunto puede traer dos efectos: uno indirecto que impacta en la mejora de la capacidad de la población para utilizar maquinarias más complejas, lo que redundará en el aumento de la producción. Otro directo, que implica que una población más preparada puede innovar más. Asimismo, defendió la idea que existen cualidades humanas que influyen en la economía del país. Entre los efectos positivos de éstas se destaca la confianza para el intercambio económico y una mejor administración de las capacidades físicas e intelectuales de los trabajadores.

Por otro lado, la influencia de los cono- cimientos o la formación en el proceso de producción de bienes fue estudiada por Say (1821) quien la denominó como industria humana. Señaló que el conocimiento es el elemento indispensable para la producción de cualquier bien e interviene en las tres fases del proceso productivo: a) estudio y conocimiento de las leyes de la naturaleza, b) aplicación de los conocimientos a un propósito útil y c) la ejecución del trabajo manual para obtener el producto, para lo que se requiere tener los conocimientos y habilidades precisas. Apuntó que el principio de que, a mayor preparación, mayor salario, no se aplica como válido cuando hace referencia a los hombres de ciencia o filósofos, porque no son los que reciben un mejor salario.

Siguiendo con la teoría de este autor, el conocimiento de los hombres de ciencia se circunscribe al primer nivel del proceso productivo, ya que este conocimiento se difunde y es transferible, lo que explicaría el fenómeno de que los países más avanzados sean quienes subvencionen a los científicos. En este sentido, Cannan (1928), refirió que los salarios no se equiparan entre las distintas profesiones ya que existen discrepancias entre los mismos, y las diferencias entre grados de preparación o habilidad requeridas por los distintos trabajos es uno de los factores de desigualdad de los ingresos que perciben los individuos.

Más adelante, Walsh (1935), planteó la decisión de las familias de asumir los gastos en educación como una decisión de inversión de capital para obtener beneficios. Su postura fue que el retorno de los beneficios de la educación es mayor que el costo de adquirirla y señaló una correlación entre los ingresos y las habilidades innatas. El autor empleó el término Capital Humano para referirse a los gastos en educación y formación en el trabajo. Esto denota una clara intención de entender la 
educación como una manera de facilitar la movilidad social y mejorar la situación económica.

Precisamente afirmando esta idea, Boudon (1977), apuntó que, si a partir de la educación no se lograba corregir la desigualdad, debían subsanarse las diferencias educativas modificando las desigualdades sociales previas. Thurow (1983) demostró que la duración del proceso formativo y la posesión de capacidades intelectuales no lograban explicar los contrastes salariales. Aunado a lo anterior, el incremento de la productividad no correspondía con el aumento de la educación de los trabajadores, ya que el mercado se rige más por la competencia por puestos de trabajo que por la competencia salarial. Thurow sostiene la existencia de un fenómeno de "sobre educación" de postulantes, y discordancia entre habilidades educativas y salarios. Parece existir una distinción de que los gastos en educación, entrenamiento, atenciones médicas, entre otros, producen Capital Humano y no físico o financiero, porque no se puede separar a una persona de sus conocimientos, salud o valores como se hace con otro tipo de activos (Becker, 1993).

Todas las anteriores aportaciones permitieron que la Teoría del Capital Humano, tuviera también acotaciones a nivel conceptual, tal como señaló Bonal (1998) el Capital Humano sentó las bases para cambios de política educativa como gasto público por niveles educativos, la existencia de lógicas diferentes para la estratificación educativa y la estratificación social, así mismo, advirtió que la educación es expresiva porque busca despertar actitudes orientadas a la disposición del aprendizaje, autonomía, comunicación, relación, entre otras.

Respecto al conocimiento, Tedesco (2000) señaló que éste es objeto de acelerados procesos de devaluación arrastrados por la velocidad del cambio tecnológico y organizacional, por lo que resulta imprescindible la educación permanente. De igual manera, apuntó que el individuo es portador de una nueva obligación que provoca la necesidad de generar en sí mismo una forma de inserción social. Brunner y Elacqua (2003) refirieron que la cantidad de la educación se desplaza por la calidad porque lo prioritario es saber cuánto se educan y con qué impacto sobre los niveles de productividad en la economía.

El Capital Humano como dueño del conocimiento es parte de los activos intangibles y más valiosos de la organización, ya que ayuda a dar forma a la estrategia, a la estructura y a los procesos de dirección que permiten lograr avances para alcanzar la competitividad. Esos son algunos de los aportes que hace el Capital Humano al desarrollo organizacional (Calderón y Mousalli, 2012; Del Canto, 2011; Sassha, 2011 y Tovar, 2017). Los componentes principales que posee el Capital Humano señalados por Blundell, Dearden, Meghir y Sianesi (2005) son: 1. la calidad temprana (cualidades con las que la persona cuenta, las cuales pueden ser adquiridas o innatas); 2. la cualificaciones y conocimientos adquiridos en la educación formal; y 3. las habilidades y conocimientos conseguidos a través de la capacitación para el trabajo. Estos autores también señalan que la falta de datos adecuados y las dificultades metodológicas han resultado en una escasez de estudios que han llevado a cabo un trabajo empírico sólido sobre el tema de los rendimientos de las inversiones de Capital Humano para los empleadores.

En la conformación de la TCH se han distinguido dos etapas importantes: La primera representa un enfoque inicialmente promovido por economistas y sociólogos, quienes buscaban tener influencia para reformar el área educativa. En la segunda etapa se confi- 
rió al Estado la función de garante de la continuidad de los estudios de la población, buscando que las personas en edad productiva, pudieran completar su formación como medio de equidad y política económica (Aronson, 2007). En ambas se percibe a la educación como un claro elemento de incidencia en la producción de riqueza y mejoramiento económico.

El Capital Humano o la calidad de la fuerza de trabajo representa uno de los factores que se asocian al crecimiento económico, ya que los atributos incorporados a los recursos humanos facilitan la creación de bienestar social y económico (OCDE, 2007). En este sentido Aronson (2007), lo concibe como un rango de conocimientos apropiados para el mejoramiento y la ampliación del rendimiento laboral. Se asocia al conjunto de bienes intangibles que intervienen en el rendimiento del capital físico (fábricas, máquinas).

En relación con la clasificación del capital de trabajo y el ajuste que eventualmente debe efectuar el individuo con su formación académica, Didier, Pérez y Valdenegro (2013) refieren que las habilidades pueden ser de carácter general (las que se asocian a los resultados de la educación formal) y de carácter específico (determinadas por el contexto en el que se desarrolla el individuo y generalmente son definidas por la organización para la que éste trabaja).

\section{Capital humano y educación ¿binomio pa- ra el éxito?}

La TCH es un enfoque de carácter exclusivamente económico y laboral y el capital físico adquiere valor en la enseñanza, como elemento principal para potenciar el desarrollo productivo del sujeto tanto en su ámbito individual como colectivo (Acevedo, 2018), de ahí que se conciba a la formación académica como un activo en el que se invierte para obte- ner ganancias a futuro.

En el marco de la globalización la $\mathrm{TCH}$ tiene una visión influyente ya que concibe a la educación como una inversión que proyecta utilidades a mediano o largo plazo (PérezFuentes y Castillo-Loaiza, 2016), y a ello se asocian directamente una serie de factores tales como crecimiento económico, calificación laboral, producción técnica, investigación, entre otros.

En la TCH se asume que la educación y los años de escolaridad están en proporción con los ingresos, el éxito económico, la empleabilidad y el aumento de la calidad de vida, sin embargo, no son los únicos, ya que requiere combinarse con políticas aplicadas en diferentes ámbitos, con el propósito de aprovechar mejor el potencial con el que se cuenta para generar mayor desarrollo social y económico (Márquez, 2017; Martínez y Lederman, 2016 y Olivera, 2017).

En ese mismo sentido, Acevedo (2018, p. 67) señala que el Capital Humano "parece omitir la existencia de elementos de orden cultural, político y social que regulan la oferta y demanda laborales". De modo que en apariencia el Capital Humano no se puede centrar únicamente en la formación académica, sino que pervive con otros componentes del contexto de los cuales el individuo no tiene control. Sin embargo, el acceso a puestos mejor calificados se relaciona directamente con elementos innatos y otros que se adquieren como la carrera cursada, el dominio de idiomas y de la tecnología e informática, e incluso el cuidado de la salud y la cultura (Anzola, 2018; Pérez-Fuentes y Castillo-Loaiza, 2016).

En sus inicios dos de los teóricos más representativos de la TCH, Becker (1993) y Schultz (1961), realizaron el planteamiento de que la posesión de educación puede ser equiparable con el usufructo de cualquier tipo de 
capital material, por lo que puede ser considerado bajo el término de rentabilidad. Bajo esta premisa se produjo una ampliación en las expectativas que se tenían depositadas en el sistema educativo, sin embargo, disminuyó su influencia cuando al pasar de los años, no se pudo asociar claramente a la rentabilidad. Aunado a esto se detectaron dos debilidades, ya que, por un lado, la educación no producía los efectos de desarrollo económico esperados y por otro, no había una relación precisa entre formación y remuneración. De esta manera se cuestionó la contribución real de las instituciones educativas, como la solución para la problemática de las marcadas diferencias sociales, aunque se logró comprobar que el esfuerzo personal funcionaba dentro de las escuelas y universidades.

Pero ese esfuerzo traducido en productividad o producto intangible, resultado de un proceso formativo mayormente longitudinal, se convierte en mercancía consumida en el mercado bajo la forma de Capital Humano. El riesgo de producción del $\mathrm{CH}$ cuando no va aparejado de desarrollo económico es la sub utilización o depreciación de la fuerza de trabajo formada en Instituciones de Educación Superior; se advierte que el riesgo de sobre producir $\mathrm{CH}$ en las universidades es la inserción en puestos de trabajo mal pagados, que redundan en una posición económica y social que provocan el descontento individual, y luego ampliado. En este contexto, el individuo debe buscar la manera de irse adaptando a las demandas del entorno a través de la adquisición de capacidades que le permitan competir en un ambiente de flexibilidad y cambio, es decir, de incrementar su capital de conocimiento, y, por ende, material (Ramírez, 2015).

Diversos autores Anzola (2018), PérezFuentes y Castillo-Loaiza (2016) y Vecino y
Zaldívar (2016) coinciden en la importancia que tiene incorporar la formación profesional y las habilidades del individuo, así como la situación laboral en sus análisis. Consideran que una persona de talento tendrá niveles de desarrollo en relación con el nivel de acumulación de conocimientos que alcance en su formación profesional, aunada a su experiencia laboral y a las cualidades innatas que posee, sumado a los valores y ética que le permiten hacer una contribución valorada en la empresa o la sociedad, indicando su potencialidad para la creación de nuevos productos o servicios.

En ese mismo sentido, aunado a la formación académica, existen cualidades inherentes al individuo desarrolladas a lo largo de su transitar por las distintas esferas vitales. Quintero (2020) señala que dos individuos en el mercado laboral, altamente competitivos en cuanto al capital académico adquirido, diferirán en rasgos como las aptitudes, actitudes, habilidades, capacidades y el compromiso con el que cada trabajador realiza las actividades en el puesto de trabajo. En este caso, uno de ellos adquiere más ventajas competitivas si muestra mejores cualidades añadidas a las competencias adquiridas por su formación profesional. Eso definitivamente constituye parte del Capital Humano.

\section{Impacto del capital humano, el caso de México}

El impacto de la Teoría de Capital Humano en la formación profesional superior es más visible en países con mejores niveles de vida, en aquellos en los que hay más ingreso per cápita y en donde el nivel de formación profesional es superior.

Organismos de alcance mundial coinciden en enfatizar la importancia del Capital Humano, tales como la Organización de las Naciones 
Unidas para la Educación, la Ciencia y la Cultura (UNESCO), quien destaca la trascendencia del conocimiento y la formación de Capital Humano como los ejes necesarios para el crecimiento de las naciones. Para lograr lo anterior, es indispensable fortalecer los sistemas de investigación e innovación, la creación de capacidad, el uso de tecnologías y las redes de científicos, la colaboración entre la universidad y el sector industrial (Organización de las Naciones Unidas para la Educación, la Ciencia y la Cultura UNESCO, 2020).

El Banco Mundial (BM), propuso medir la posición de los países con base en una serie de indicadores que están integrados en cuatro dimensiones: 1. funcionamiento del régimen de incentivos económicos, 2. nivel de desarrollo de la educación y el Capital Humano, 3. capacidades de innovación y 4. uso de Tecnologías de la Información. Ese organismo refiere que México junto a Chile, Colombia, España, Perú y Portugal, han mostrado una trayectoria de progreso, sin embargo, todavía existe un reducido avance hacia la economía global debido a diversos y complejos problemas a los cuales deben enfrentarse todos los países (Banco Mundial, 2020).

Diversas situaciones frenan el desarrollo de las naciones subdesarrolladas, sin embargo, el que resulta más negativo, es el asociado a la inversión que se realiza en la educación superior. Domínguez (2015) señaló que, en el mundo globalizado, los beneficios y logros de los avances son desiguales para las naciones y los grupos sociales, ya que la diferencia que existe entre las naciones desarrolladas, en desarrollo y subdesarrolladas, se debe en gran medida a los recursos que permiten impulsar y mejorar la calidad de la ciencia y la tecnología que despliegan.

En ese mismo sentido, reconociendo la simbiosis del Capital Humano y las instituciones educativas para el desarrollo de las nacio- nes, Brunner y Ganga (2016, p. 22) señalan que, para abordar adecuadamente las tareas de crecimiento y competitividad, así como superar la pobreza, las sociedades necesitan incrementar de forma sostenida su Capital $\mathrm{Hu}-$ mano, tarea en la cual las IES tienen un rol decisivo. Apuntan a que la participación efectiva de Latinoamérica en la geopolítica del conocimiento avanzado, así como del gasto público y privado en $\mathrm{I}+\mathrm{D}$, son extraordinariamente débiles. Este fenómeno ocurre casi en cualquiera dimensión, en la que intervenga el Producto Interno Bruto (PIB).

Ante el enorme reto de tener finanzas cada vez más escasas, para el caso de México, lo que se necesita, es reconocer que la instrumentalización de la educación como un servicio, permitirá el acceso a un mercado de oportunidades laborales, y a mejores condiciones socioeconómicas; otro enorme desafío que se presenta, es garantizar la igualdad de oportuni -dades en el acceso a la Educación Superior, la cual debe ser accesible a todos los ciudadanos que tengan la motivación para estudiar y estén dispuestos a asumir los esfuerzos personales que implica; para lo cual, las IES deben optimizar los recursos financieros disponibles y diseñar estrategias de captación de recursos menos dependientes de la financiación pública y de las matrículas que deben pagar los estudiantes (Martí-Noguera, Licandro y Gaete -Quezada, 2018).

El Instituto Nacional para la Evaluación de la Educación (INEE, 2018), refiere que para el ciclo escolar 2017-2018, la mayor tasa neta de cobertura la presentó la educación primaria con $98.5 \%$, le siguieron la educación secundaria con $84.3 \%$, la educación preescolar con $71.6 \% \mathrm{y}$, por último, la educación media superior con $63.8 \%$. Se observa un decremento considerable de las cifras en función del grado de avance que van teniendo los estudiantes en los diversos niveles académicos. 
Las ideas anteriores prevalecen entre los teóricos que estudian a la educación desde el enfoque económico, por ejemplo, Schultz (1961), definió al Capital Humano como aquel que incluye elementos de tipo cualitativo: habilidades, conocimientos y en general todos aquellos atributos que tienen injerencia sobre la capacidad individual y que permiten realizar el trabajo productivo. Se espera que la inversión que se efectúe para mejorar las capacidades del individuo, aumentarán el valor de la productividad del trabajo y producirán un rendimiento positivo.

Vecino y Zaldívar (2016) analizan ampliamente de los conceptos asociados al Capital Humano, y refieren que éste es el portador del conocimiento, por lo que debe estar contemplado en la expresión de valor de los productos y servicios, por tener una responsabilidad fundamental como diferenciador del trabajo abstracto y del concreto, como del complejo y del simple. Así mismo, es aquel valor en forma de conocimientos técnicocientíficos, culturales y éticos, que puede acumular una persona de forma independiente o agrupados en equipos de trabajo en una empresa o en la sociedad con el propósito de darle valor al objeto de trabajo.

La inversión en Capital Humano en México resulta sumamente importante, según el Consejo Nacional de Ciencia y Tecnología (CONACYT), aunque reconoce que no se puede soslayar que ésta debe ir acompañada de una estrategia de desarrollo acertada, en aras de disminuir las brechas de desigualdad entre entidades federativas, en términos de capacidades científicas y tecnológicas, creación de infraestructura y fomento a la vinculación entre los sectores académico, privado y gubernamental (CONACYT, 2020).

Diversos tópicos relacionados con el Capital Humano han sido motivo de debate, como los señalados por Kido y Kido (2015) quienes refieren que aún existen lagunas entre la eficacia de la educación formal como factor para aumentar la productividad individual y el crecimiento económico de un país. El análisis que efectúan consiste en que al ser el Capital Humano una variable difícil de medir, en ocasiones se emplean los años promedio de escolaridad como referente y si en la TCH se dice que una mayor educación genera gente más productiva y un aumento en el ingreso de las personas, se presupondría entonces una nación más productiva y un aumento del ingreso nacional. Concluyen señalando con base en su estudio, que existe evidencia empírica de que el Capital Humano presenta la mayor explicación en el comportamiento entre la relación de escolaridad e ingreso personales en México.

Por otro lado, desde una perspectiva humana, considerando las connotaciones que para los empleadores tiene el Capital Humano y la exigencia de mayores grados académicos, Ramírez (2015) cuestiona el hecho de que, a través del Capital Humano, exista una versión renovada del espíritu del capitalismo hacia el hombre que cuente con conocimientos, habilidades y un estado de salud apropiado, ya que puede participar en los procesos de producción y generar ganancias. Es por ello que, al aplicarse esta voluntad a los seres humanos, al vérseles como capital, el empresario va a la búsqueda de obtener de los trabajadores el rendimiento máximo posible, sin importar si estos trabajan con el conocimiento o con la fuerza física. Además, cada día se requieren mayores niveles de educación y habilidades para acceder a los puestos de trabajo.

En México la educación se ha desarrollado bajo el enfoque de Capital Humano, al buscar incrementar las posibilidades de producción de las personas, sin embargo, no fue 
acorde al enfoque político y social del neoliberalismo ya que favorece los títulos de posgrado, pero la producción académica de las personas que los obtienen, es limitada (Castillo y Karam, 2015). El Capital Humano considera a las personas por su productividad y desempeño profesional, pero se dejan fuera aspectos tales como la libertad de decisión, los valores y el bienestar de las personas.

\section{Conclusiones}

Existen múltiples retos y limitaciones que los países del mundo deben enfrentar y superar, incluido México, en materia de Capital $\mathrm{Hu}-$ mano para el mejoramiento social y económico. Algunos llegan a convertirse en una espiral de la cual resulta difícil salir. La superación de estos retos se orienta hacia la imperante necesidad de las naciones de considerar como un área de oportunidad la formación de sus estudiantes en todos los niveles educativos. Para el caso particular de México, es necesario fortalecer la enseñanza de matemáticas, lectura y ciencias desde los niveles básicos de educación.

Basada en el principio de que una población más preparada puede innovar más, prevalece la idea que, al elevarse la calidad de los distintos niveles académicos, se esperaría un incremento en la generación de patentes, registros de derechos de autor, desarrollo de proyectos de investigación, por mencionar solo algunos indicadores que inciden directamente en la economía del conocimiento de las naciones.

En México, sigue presentándose uno de los problemas que Say identificó desde 1821, y es el relativo a que los hombres de ciencia no son los que reciben un mejor salario, así como que no hay mejores salarios para los más altos niveles de preparación. Es indispensable revalorar y remunerar mejor el trabajo del Capital Humano que se dedica al desarrollo de la ciencia, la tecnología y la innovación. De este modo se esperaría que aumente el número de personas interesadas en estos desarrollos. Lamentablemente, en países como México existe el fenómeno referido por Thurow (1983) relativo a la existencia de la "sobre educación" de postulantes, y discordancia entre habilidades educativas y salarios, los cuales son cada vez más exiguos.

Otro de los retos a enfrentar por parte de la formación de Capital Humano es la relativa a la calidad de la educación que se imparte, pero con la incorporación del componente del impacto cualitativo que ésta tiene sobre la productividad y el desarrollo económico. Es urgente realizar estudios que permitan entender mejor las relaciones entre educación y las variables enunciadas. Por otro lado, es indispensable optimizar la administración el erario y dirigir los esfuerzos a incrementar la inversión que se hace en innovación y desarrollo tecnológico, para cumplir a cabalidad con la disminución de brechas que existen en todo el país.

Las organizaciones de toda índole deben dejar de visualizar a sus trabajadores de forma un tanto genérica y proceder a diseñar planes de crecimiento cuantitativos y estandarizados con el propósito de que sus colaboradores se sientan incentivados en mejorar la formación, conocimientos y habilidades con los que cuentan, con la certeza de que esto les permitirá escalar a mejores ingresos.

En ese mismo sentido, el paso siguiente debiera ser diseñar planes de crecimiento de vida, enfocados a la formación permanente, a lo largo de toda la vida de las personas, en los que puedan tener opción a la mejora constante de sus condiciones en el contexto familiar, organizacional y social. 
Para dar muestra de algunos ejemplos de lo aquí descrito, la UNESCO (2018), a través de la Unidad de Aprendizaje a lo Largo de Toda la Vida, propuso seis características específicas con las que deben contar las ciudades para ser denominadas "Ciudades de Aprendizaje": 1) promover el uso de recursos para el aprendizaje inclusivo desde la educación básica a la superior, 2) revitalizar el aprendizaje en las familias y comunidades, 3) facilitar el aprendizaje para el trabajo y en el lugar de trabajo, 4) extender el uso de tecnologías modernas de aprendizaje, 5) mejorar la calidad y excelencia en el aprendizaje y 6) fomentar una cultura del aprendizaje a lo largo de toda la vida.

El gobierno de Nuevo León promovió el proyecto "Monterrey ciudad internacional del conocimiento", a través de la creación de una región en la cual las empresas, centros de investigación y universidades pudieran innovar y de esta forma, lograr ventajas competitivas para el estado. Se busca, además, mayor efectividad por sus ciudadanos y organizaciones con el objetivo de promover el desarrollo económico y social de la propia comunidad basado en la innovación (Villareal, 2009). Derivado de este esfuerzo se construyó el Parque de Investigación e Innovación Tecnológica (PIIT), el cual alberga 35 centros de investigación, públicos y privados, en donde convergen investigadores, científicos y tecnólogos, así como la industria (González, 2020).

\section{Referencias}

Acevedo, M. (2018). La teoría del Capital Humano, revalorización de la educación: análisis, evolución y críticas de sus postulados. Revista Reflexiones y Saberes, 5(8), 58-72. Recuperado de https://cutt.ly/AfJheH2

Anzola, O. (2018). La gestión de recursos humanos y la construcción de subjetividades en las nuevas condiciones de la economía. Universitas Psychologica, 17(1), 1-14. doi:10.11144/ Javeriana.upsy17-1.grhe

Aronson, P. (2007). El retorno de la teoría del Capital Humano. Fundamentos en humanidades, 8(), 9-26. Recuperado de https://cutt.ly/1 fJkUF6

Banco Mundial [BM]. (2020).¿Qué hacemos? Recuperado de http://www.bancomundial.org/es/what -we-do

Becker, G. (1993). Human Capital. A Theoretical and Empirical Analysis with Special Reference to Education (3a ed.). New York: The University of Chicago Press.

Blundell, R., Dearden L., Meghir, C. y Sianesi, B. (2005). Human Capital Investment: The returns from education and training to the individual, the Firm and the Economy. Fiscal Studies, The Journal of Applied Public Economics, 20(1), 1-23. doi:10.1111/j.14755890.1999.tb00001.x

Bonal, X. (1998). Sociología de la educación. Una aproximación crítica a las corrientes contemporáneas. Barcelona: Paidós.

Boudon, R. (1977). Effets pervers et ordre social. Paris: Presses Universitaires de France.

Brunner, J. y Elacqua, G. (2003). Informe Capital Humano en Chile. Chile: Universidad Adolfo Ibáñez.

Brunner, J. y Ganga, F. (2016). Dinámicas de transformación en la educación superior latinoamericana: Desafíos para la gobernanza. Opción, 32 (80), 12-35. Recuperado de https:// cutt.ly/8fJh $8 \mathrm{CN}$

Calderón, J. y Mousalli, G. (2012). Capital Humano: elemento de diferenciación entre las organizaciones. Revista Actualidad, 25(24), 5-18. Recuperado de https://cutt.ly/afJknpZ

Cannan, E. (1928). Wealth. A brief explanation of the causes of economic welfare. Londres: Staples Press.

Castillo, Y. y Karam, M. (2015). Capacidad humana vs Capital Humano: desde dónde enfocar la educación. Dilemas Contemporáneos: Educación, Política y Valores, 2(2), 1-18. Recuperado de https://cutt.ly/3fJleah

Consejo Nacional de Ciencia y Tecnología [CONACYT]. (2017). Informe General del Estado de la Ciencia, la Tecnología y la Innovación. México 2016. Recuperado de https:// cutt.ly/qfJky1c

Consejo Nacional de Ciencia y Tecnología 
[CONACYT]. (2020). Sistema Nacional de Investigadores. Recuperado de: http:// www.conacyt.mx/index.php/el-conacyt/ sistema-nacional-de-investigadores.

Del Canto, E. (2011). Gestión del Capital Humano, competencias y sociedad del conocimiento. Observatorio laboral, 4(8), 89-113. Recuperado de https://cutt.ly/tfJkmyP

Didier, N., Pérez, C. y Valdenegro, D. (2013). Capacitación y Capital Humano: análisis de las últimas dos décadas. Revista de Psicología, 22 (2), 87-99. Recuperado de https://cutt.ly/ DfJkIMK

Domínguez, S. (2015). Diez razones para ser científicos. Perfiles Educativos, 37(150), 210-215. Recuperado de https://cutt.ly/3fJlaEl

González, A. (2020). Nace el Monterrey Digital Hub sin la participación del Gobierno del Estado. Milenio 2020. Recuperado de https://cutt.ly/ qfJzr6r

Instituto Nacional para la Evaluación de la Educación [INEE] (2018). Tasa neta de cobertura. Recuperado de https://cutt.ly/1 fJg0wJ

Kido, A. y Kido, M. (2015). Modelos teóricos del Capital Humano y señalización: un estudio para México. Contaduría y Administración, 60(4), 723-734. Recuperado de https://cutt.ly/ NfJlq93

Labraña, J., Brunner, J. y Álvarez, J. (2019). Entre el centro cultural y la periferia organizacional: la educación superior en América Latina desde la Teoría de Sistemas-mundo de Wallerstein. Estudios públicos, 156, 107-141. Recuperado de https://cutt.ly/dfJj2Mp

Márquez, A. (2017). Educación y desarrollo en la sociedad del conocimiento. Perfiles Educativos, 39(158), 3-17. Recuperado de https:// cutt.ly/RfJgM1Y

Martínez, A. y Lederman, N. (2016). ¿Paradoja del progreso o de la decadencia? Retornos en la educación y brechas salariales en Venezuela: 1998-2012. Revista Gaceta Laboral, 22(3), 187 - 206. Recuperado de https://cutt.ly/ wfJkKgw

Martí-Noguera, J., Licandro, O. y Gaete-Quezada, R. (2018). La Responsabilidad Social de la Educación Superior como bien común. Concepto y desafíos. Revista de la Educación Superior, 47(186), 1-22. doi:10.36857/ resu.2018.186.353

Olivera, I. (2017). Las potencialidades del proyecto educativo de la Universidad Veracruzana Intercultural: una crítica al desarrollo desde la noción del Buen vivir. Revista de la Educa- ción Superior, 46(181), 19-35. https:// doi.org/10.1016/j.resu.2016.08.004

Organización de las Naciones Unidas para la Educación, la Ciencia y la Cultura [UNESCO]. (2018). Comunidades en acción: aprendizaje a lo largo de toda la vida para el desarrollo sostenible. Recuperado de https://cutt.ly/ XfJlZ3Y

Organización de las Naciones Unidas para la Educación, la Ciencia y la Cultura [UNESCO]. (2020).¿Qué es la UNESCO? Recuperado de http://www.unesco.org/new/es/unesco/aboutus/who-we-are/introducing-unesco/

Organización para la Cooperación y Desarrollo Económico [OECD] (2007). La Educación Superior $y$ las Regiones: Globalmente Competitivas, Localmente Comprometidas. doi:10.1787/9789264064690-es

Pérez-Fuentes, D. y Castillo-Loaiza, J. (2016). Capital humano, teorías y métodos: importancia de la variable salud. Economía Sociedad y Territorio, (52), 651-674. doi:10.22136/ est0522016675

Quintero, W. (2020). La formación en la teoría del Capital Humano: una crítica sobre el problema de agregación. Revista Análisis Económico, 35(88) 239-265. Recuperado de http:// analisiseconomico.azc.uam.mx/index.php/rae/ article/view/519

Ramírez, D. (2015). Capital Humano: una visión desde la teoría crítica. Cuadernos EBAPE.BR, 13, (2), 315-331. Recuperado de https://cutt.ly/ $\mathrm{SfJkCOb}$

Sassha, A. (2011). ¿Es posible medir el impacto del Capital Humano en los resultados de la organización?: el rol de recursos humanos, modelo de medición y otros indicadores del Capital Humano. Revista Perspectivas, 27(), 113-129. Recuperado de https://cutt.ly/LfJkmBb

Say, Jean-Baptiste. (1821). A treatise on political economy of the production, distribution and consumption of wealth. (6a, ed.) New York: Agustus M. Kelly.

Schultz, T. (1961). Investment in Human Capital. American Economic Review, 51(1), 1-17

Senior, N. (1836). An outline of the science of political economy. Reprint of economic classics. New York: Augustus, M. Kelley Bookseller

Stuart-Mill, J. (1864). On liberty. Londres: Longman, Green, Longman Roberts \& Green.

Tedesco, J. (2000). Igualdad de oportunidades y política educativa. Cadernos de Pesquisa, 34 (123), 555-572. Recuperado de https://cutt.ly/ nfJkv72 
Thurow, L. (1983). Educación e igualdad económica. Revista Educación y Sociedad, 2(), 157-171. Recuperado de https://cutt.ly/CfJkcsP

Tovar, B. (2017). La teoría del Capital Humano llevada a la práctica en las ciudades de aprendizaje. Nóesis, 26(51), 45-56. Recuperado de https:// cutt.ly/DfJkQ1C

Vecino, F. y Zaldívar, A. (2016). El Capital Humano en la transformación de la estructura y la superestructura social. Dilemas Contemporáneos: Educación, Política y Valores, 4(1), 123. Recuperado de https://cutt.ly/4fJk5P7

Villareal, R. (2009). Monterrey, ciudad internacional del conocimiento. Comercio exterior, 59(11), 873-885. Recuperado de https:// cutt.ly/5fJINPs

Walsh, J. (1935). Capital concept applied to man. The Quarterly Journal of Economics, 49(2), 255285. doi: $10.2307 / 1884067$ 